Revista de Psicología Vol. 36 (1), 2018 (ISSN 0254-9247)

\title{
Actitud y resistencia al cambio organizacional en trabajadores mineros
}

\author{
Jorge Salgado Roa ${ }^{1}$, Francisco Lería Dulčiće, Lina Arcos C. ${ }^{3}$, \\ Cynthia Pineda A. ${ }^{4}$, Claudia González C. ${ }^{5}$ \\ Universidad de Atacama-Chile
}

El objetivo del estudio fue analizar la relación entre la actitud y resistencia al cambio organizacional en trabajadores mineros. Se utilizó una estrategia asociativa de tipo transversal de diseńo de grupos naturales y la escala de actitudes hacia el cambio (EAC), la escala de la resistencia al cambio (ERC); y una encuesta para recabar datos sociodemográficos. Participaron 251 trabajadores. Los resultados indican una actitud favorable hacia el cambio organizacional, y un nivel medio/moderado de resistencia. Se presentan diferencias significativas en las puntuaciones de la ERC y la EAC en función de la faena laboral. La jerarquía laboral genera un efecto moderado en las diferencias de los factores asociados a la responsabilidad y la actitud de cinismo al cambio. Con relación a la edad, los trabajadores mayores de 51 ańos puntúan más bajo que los rangos menores en la dimensión actitud de temor hacia el cambio. Palabras claves: Actitud al cambio, resistencia al cambio, cambio organizacional, trabajadores mineros, factores organizacionales

\section{Attitude and resistance to organizational change in mining workers}

The aim of the study was to analyze the relationship between attitude and resistance to organizational change in mineworkers. An associative transversal strategy of natural groups design was used. The following instruments were administered: the scale of attitudes towards change (EAC), the scale of resistance to change (ERC) and a survey to collect socio-

1 Doctor en Ciencias de la Educación. Docente y subdirector del Departamento de Psicología de la Universidad de Atacama. Dirección postal: Av. Copayapu 485, Copiapó, Chile. Contacto: jorge.salgado@uda.cl

2 Magíster en Psicología y docente del Departamento de Psicología de la Universidad de Atacama. Dirección postal: Av. Copayapu 485, Copiapó, Chile. Contacto: francisco.leria@uda.cl

3 Licenciada en Psicología de la Universidad de Atacama. Dirección postal: Av. Copayapu 485, Copiapó, Chile. Contacto: Lina.arcos@pucobre.cl

4 Licenciada en Psicología de la Universidad de Atacama. Dirección postal: Av. Copayapu 485, Copiapó, Chile. Contacto: Cynthia.pinedaaa@gmail.com

5 Licenciada en Psicología de la Universidad de Atacama. Dirección postal: Av. Copayapu 485, Copiapó, Chile. Contacto: clau.marcela.gonzalez@gmail.com 
demographic data. 251 workers participated, mostly men. The results indicate a favorable attitude toward organizational change, and a medium/moderate level of resistance. Significant differences in scores of ERC and EAC according to the work task were found. Labor hierarchy generates a modest effect on differences in factors associated with responsibility and the attitude of cynicism to change. With respect to age, workers older than 51 years of age scored lower than the lowest ranks in the dimension of fearful attitude towards change. Keywords: Attitude change, resistance to change, organizational change, mineworkers.

\section{Atitude e resistência à mudança organizacional em trabalhadores de mineraçáo}

O objetivo do estudo foi analizar a relação entre a ação e a resistência ao cambio organizaçional em mineiros. Utilizou-se uma estratégia asociativa de tipo transversal de planejamento de grupos naturais midimdo-se con uma escada de atitudes ao cambio (EAC), a escada da resistência ao cambio (ERC); e uma pesquisa para coletar dados socio-demográficos. Participaram 251 trabalhadores. Os resultados indicam uma atitude favorável para o cambio organizacional e um nível médio / moderado de resistência. Foram apresentadas diferenças significativas na classificação da ERC e da EAC em função da categoría do trabalho feito. A hierarquia laboral gera um efeito moderado nas diferenças dos fatores associados à responsabilidade e à atitude de cinismo ao cambio. Con relaçáo a idade, os trabalhadores maiores de 51 anhos tiveron uma contagem superior aos das faixas etáreas menores na dimensão da atitude de temor para o cambio.

Palavras-chave: Atitude para mudar, resistência à mudança, mudança organizacional, trabalhadores mineiros, fatores organizacionais.

\section{Attitude et résistance au changement organisationnel chez les travailleurs miniers}

L'objectif de l'étude était d'analyser la relation entre l'attitude et la résistance au changement organisationnel chez les travailleurs miniers. Nous avons utilisé une stratégie associative de conception transversale des groupes naturels et l'échelle des attitudes envers le changement (EAC), l'échelle de la résistance au changement (ERC); et une enquête pour collecter des données sociodémographiques. 251 travailleurs ont participé. Les résultats indiquent une attitude favorable envers le changement organisationnel et un niveau de résistance moyen / modéré. Il existe des différences significatives dans les scores de l'EAC et de l'ERC en fonction de la journée de travail. La hiérarchie du travail génère un effet modéré dans les différences des facteurs associés à la responsabilité et l'attitude du cynisme à changer. En ce qui concerne l'âge, les travailleurs de plus de 51 ans obtiennent des scores inférieurs à ceux des groupes d'âge inférieurs dans l'attitude de peur face au changement.

Mots-clés: attitude envers le changement, résistance au changement, changement organisationnel, travailleurs miniers, facteurs organisationnels. 
Entre los fenómenos más observados al interior de las organizaciones contemporáneas, y además propio a las repercusiones que conllevan los procesos globalizantes, se encuentra la generación de ambientes laborales competitivos y de alta exigencia, en los cuales los trabajadores se hallan sometidos a permanentes exigencias, cambios e incertidumbres. La globalización de los mercados, la difusión de la tecnología de la información y redes sociales; y los cambios en la naturaleza de la fuerza laboral, repercuten en los procesos de transformación armónica de las organizaciones y sus miembros (López, Restrepo \& López, 2013). Los especialistas han enfatizado que estas presiones y contingencias del contexto socioeconómico han generado la necesidad de responder con elementos no solo tecnológicos y de innovación, sino también actitudinales, particularmente de flexibilidad y apertura al cambio, como una condición sine qua non al funcionamiento y desempeño del trabajador en la organización contemporánea (Rodríguez, 2011). Sin embargo, la evidencia sugiere la adaptabilidad, flexibilidad y enfrentamiento al cambio al interior de las organizaciones son una de las variables más resistidas por los trabajadores (DiFabio \& Gori, 2016).

\section{El cambio organizacional}

Existe un amplio espectro de investigaciones respecto del cambio organizacional desde diversas perspectivas teóricas, variables involucradas, métodos de estudio y actores involucrados (Ferrer, 2015; Lee, Weiner, Harrison \& Belden, 2013; Pérez, Vilariño \& Ronda, 2016, Rodríguez \& Mladinic, 2016; Romero, Matamoros \& Campo, 2013, Schaffer, Sandau \& Diedrick, 2013). El cambio organizacional se ha definido como un proceso observable por el cual las organizaciones pasan de un estado actual a algún estado futuro, generalmente con el propósito de incrementar su efectividad, a través de la adaptación a las 
nuevas contingencias a las cuales se enfrentan: por ejemplo, transformaciones internas, necesidad de actualización e innovación y/o adquisición de nuevos aprendizajes para sus miembros (Chiavenato, 2009); incluye un estado constante de alteración, variación o modificación de la organización y sus miembros (Acosta, 2003); y puede ser precipitado por cualquier modificación que se de en el entorno. Se puede definir globalmente como la modificación observada en la estructura de un sistema organizacional (Porret, 2010), incluye la modificación y/o alteración que ocurre en el entorno de trabajo, estructurales y funcionales tanto como del comportamiento de sus miembros y corresponde a uno de los fenómenos más relevantes que ocurren al interior de las organizaciones contemporáneas (García, Gómez \& Londoño, 2009).

El cambio organizacional ha sido categorizado con tres componentes básicos: 1) el contexto, 2) el contenido, y 3) el proceso (García et al., 2008). Otros enfoques lo han subdividido en seis dimensiones (García, Rubio \& Bravo, 2007): a) cambios dentro del sistema, al sistema y de sistema, b) carencia-oportunidad, c) adaptación-transformación, d) estructuras-fluidos e) continuidad-discontinuidad; y f) pensamiento lineales-sistémicos.

El estudio respecto de las variables que inciden en la ocurrencia del cambio al interior de las organizaciones, han sido atribuidas a diversos contextos y dimensiones. Por ejemplo, una clasificación indica el cambio organizacional ocurre debido a causales y desencadenantes externas e internas, donde las variables externas comprenden las tendencias que son impuestas a la organización desde afuera (ej.: los mercados actuales, los consumidores y/o las innovaciones tecnológicas); y las variables internas, ligadas a la estructura y estrategias al interior de la organización (Sandoval, 2014). Otra clasificación corresponde a las variables superficiales y profundas (Benoit \& Meston, 1995); donde las primeras abarcan cambios que son fáciles de implementar y de bajo costo; sin limitaciones a su cantidad y que — sin perjuicio- pueden terminen en cambios más profundos. Consecuentemente, los cambios profundos o de segundo orden, poseen una mayor influencia en la organización y cultura organizacional y su principal característica es 
la dificultad para ser generados a consecuencia del profundo impacto que tienen en los trabajadores, las demandas de tiempo que implican y los recursos que estos exigen en las diferentes áreas de la organización. Finalmente, otras clasificaciones del cambio organizacional aluden a las dimensiones deductivas e inductivas; de carácter continuo o episódico; evolutivos o acumulativos; emergentes o no intencionados; ocasionales, contingentes y de aparición infrecuente en el tiempo; entre otros (García et al., 2008; Porret, 2010; Quirant \& Ortega, 2006).

De acuerdo con Chiavenato (2004), para el logro de un cambio al interior de la organización se debe primariamente modificar el set mental de los miembros de la misma, así, se hace indispensable preparar el ambiente psicológico para que sea adecuado y propicio a la trasformación esperada. La investigación en este sentido ha destacado algunas de las variables psicológicas que juegan un papel en la ocurrencia del cambio al interior de las organizaciones, por ejemplo, los sujetos que muestran una mayor tendencia a la aceptación del cambio, resuelven más fácilmente las demandas que los ambientes laborales les imponen (DiFabio \& Gori, 2016); poseen mayores niveles de bienestar personal (DiFabio \& Bucci, 2015); y/o demuestran habilidades blandas más adaptativas (DiFabio \& Saklofske, 2014; DiFabio \& Kenny, 2015). Otras investigaciones señalan la consciencia y apresto al cambio posee un impacto en el bienestar del trabajador durante los procesos de cambio e inestabilidad organizacional (Pahkin et al., 2014); y/o las disposiciones individuales de los trabajadores al mismo lo modulan ampliamente (Dennis, Erwin \& Garman, 2010). La apertura al cambio organizacional se ha visto relacionada también a mejores niveles de inteligencia emocional (Sánchez \& Nguyen, 2009); y se ha constatado que la percepción subjetiva de la relación entre jefaturas y trabajador es una variable crucial en la promoción del cambio (Van Dam, Oreg \& Schyns, 2008).

\section{La resistencia al cambio en la organización contemporánea}

El comportamiento individual de los miembros de una organización y como estos se resisten al cambio e innovación es una de las áreas más 
estudiadas y con una mayor validación empírica en la literatura científica (Johansson, Kauffeldt, Helldin \& Carlström, 2014; Zuinaga de Mazzei, 2014). Los especialistas han enfatizado que las reacciones iniciales propias y de carácter muchas veces emocional de los trabajadores son normales y predecibles, debido a la modificación de los comportamientos y rutinas que hasta el momento les proporcionaban comodidad y seguridad (Marshak, 2007). Además, el cambio organizacional les exige: "un esfuerzo, intelectual o físico provoca inquietud, incertidumbre, temores, desconfianza y sensación de riesgo" (Porret, 2010, p. 32). Las dinámicas de cambio y resistencia al mismo se han circunscrito al conflicto ineludible que tiene lugar entre la cabeza de la organización y los miembros de la misma, los cuales finalmente tienen la responsabilidad final de llevarlo a cabo (Macrì, Tagliaventi \& Bertolotti, 2002).

Una de las áreas de mayor importancia en la investigación del cambio y resistencia al mismo, aborda la identificación de las variables psicológico-actitudinales del trabajador, incluyendo lo cognitivo y lo afectivo, existiendo una amplia evidencia empírica al respecto (Aldana de Becerra \& Joya, 2011; Contreras \& Barbosa, 2013; García, 2005; García et al., 2011; Montealegre \& Calderón, 2007; Sagredo, 2012; Sandoval, 2014). Por ejemplo, Giangreco y Peccei (2005 en Erwin \& Garman, 2010, p. 42); plantean que la resistencia al cambio se manifiesta en polaridades como rasgo psicológico, desde la pasividad a la apertura y/o desde el apoyo a la resistencia. Oreg (2006), sugiere que el nivel de entendimiento del cambio tiene una influencia en los sentimientos el sujeto hacia el mismo, además de comprometer su capacidad para iniciarlo y mantenerlo en el tiempo. En la misma línea, Chreim (2006) sugiere que las reacciones ante el cambio dependen de la autopercepción de sujeto de las habilidades que le son necesarias para enfrentarlo, junto a diversas reacciones emocionales, que ocurren simultáneamente, tales como: la euforia, ansiedad, ira, miedo, entusiasmo, aprehensión y estrés.

Otros estudios señalan a las variables neuroticismo y autoconciencia, como claves y mutuamente excluyentes en la capacidad del trabajador de afrontar exitosamente la relación dialéctica resistencia-proclividad 
y apertura al cambio organizacional (Foldal, Langvik, Saksvik-Lehouillier, 2016); y/o la actitud disposicional del contrato psicológico previo a un proceso interno transformativo de la organización, entre liderazgo y trabajadores de una empresa, incide en la disposición y efectividad final del cambio organizacional (García-Rubiano \& Forero-Aponte, 2015). Por otra parte, el estudio de las variables comportamentales ha mostrado que la resistencia al cambio posee un componente de disentimiento manifestado, visible en actitudes de — por ejemplo- bajo esfuerzo por el mismo, ausencia de cooperación activa, no participación en su promoción y ausencia de esfuerzo por comunicar a otros las razones y motivos del mismo (Erwin \& Garman, 2010). Los mismos autores señalan que la participación en el proceso de cambio está asociada a la percepción positiva del mismo. Por último, la evidencia empírica al respecto sugiere que cuando los miembros de una organización perciben que deben cambiar de una actividad a otra, la productividad tiende a decrecer, percibiéndose como amenazante, y por lo tanto, se manifiesta la tendencia a evaluar la contingencia de cambio en forma negativa (Tornay \& Milán, 2001).

Al hablar de actitud de resistencia al cambio, los especialistas han precisado que las personas manifiestan ante el mismo, modos actitudinales y disposiciones diversas. Estas disposiciones son definidas por Gil y Caccioppo (2007), como: "la percepción evaluativa, general y duradera, sobre alguna persona, objeto o problema” (p. 302); poseyendo un carácter actitudinal, aprendido y persistente en el tiempo (Cloninger, 2003). Tienen una naturaleza aprendida y comprenden la respuesta a un objeto o situación, favorable o desfavorablemente, apuntando a los sentimientos positivos o negativos del sujeto respecto de su conducta en cuestión (Stefani, 2005). Otro autor, Furnham (2001), al respecto señala que las reacciones más frecuentes al cambio al interior de la organización son evitativas, incluyendo: la deserción, resistencia activa, oposición, resignación, aceptación con reserva y apoyo activo; y las principales causas asociadas a la resistencia al cambio se han contado en una serie de factores contribuyentes. Otros autores incorporan un gran número de condiciones y detonantes de una actitud de resistencia al 
cambio, como por ejemplo: el miedo a lo desconocido; la falta de información; los factores históricos; las amenazas al estatus; las amenazas de los expertos; las amenazas al pago y perdida de otros beneficios; la baja confianza organizativa; la reducción en la interacción social; el miedo al fracaso; la poca flexibilidad organizativa; el aumento de las responsabilidades laborales; la disminución en las responsabilidades laborales; el temor a no poder aprender las nuevas destrezas requeridas, entre muchas otras (Lefcovich, 2006).

De acuerdo con King (2003, en García, Rojas \& Díaz, 2011, p. 130) se han identificado cuatro teorías principales acerca de la resistencia al cambio organizacional: 1) la resistencia como respuesta inevitable del comportamiento; b) la resistencia como insurrección y lucha de clases de tipo político; c) la resistencia como contrapeso constructivo; y, d) la resistencia como una reestructuración cognitiva y cultural.

Estudios realizados en Chile en torno a la resistencia al cambio organizacional revelan que los aspectos de coordinación frente a dinámicas de cambio impuesto interceden en la calidad del mismo, siendo por el contrario la descoordinación un factor generador de resistencia (Ossandón, 2013); lo que se muestra en concordancia con las investigaciones internacionales en torno al papel de la comunicación en la facilitación del cambio organizacional (Petrou, Demerouti \& Schaufeli, 2016; Zuinaga de Mazzei, 2014). Otros interesantes hallazgos se relacionan en torno a la ambivalencia actitudinal en situaciones de premura por el cambio, en la cual trabajadores expuestos a información inconsistente, presentan niveles mayores de ambivalencia y resistencia, evidenciando que la variable ambivalencia es un factor moderador de la relación entre actitud e intención conductual del trabajador por el cambio (Rodríguez \& Mladinic, 2016).

\section{La organización en el contexto de la minería chilena en la región de Atacama de Chile}

Chile ha sido conocido por su minería y su amplia capacidad tecnológica y organizativa en torno a la misma. Tradicionalmente ha sido un sector de alta importancia para el país por su contribución al 
producto bruto interno (PBI) nacional. Mientras en la década de 1960 recién alcanzaba un $8 \%$ del $\mathrm{PBI}$ en términos reales, hacia principios del año 2000 llegó a un 20\%, para bajar a un 12\% al 2012. En el caso de la región de Atacama la actividad minera al mismo representó más de un 40\% del PBI regional, según la Comisión Chilena del Cobre (Comisión Chilena del Cobre [Cochilco], n/d).

La mediana minería del cobre en la cual se sitúa la empresa seleccionada para este estudio, alcanza una producción anual al año 2014 de 38 mil toneladas de cobre fino, mostrando utilidades para la empresa de US\$36 millones, un 33\% inferior al año anterior. Tiene una dotación de 761 trabajadores de plantas los cuales se distribuyen en 12 ejecutivos, 13 superintendentes, 194 supervisores y profesionales; 542 operadores, técnicos y administrativos. También proporciona trabajo a varias empresas contratistas. Desarrolla sus operaciones en la Comuna de Tierra Amarilla, región de Atacama Chile, desde el año 1989, y pertenece a capitales chilenos. La empresa esta predominantemente dedicada a la exploración y explotación de recursos minerales, preferentemente de cobre, con la máxima seguridad y tecnología, con el fin de proteger a sus trabajadores, la comuna y el medio ambiente. Genera empleo en la comuna de Copiapó y Tierra Amarilla a 761 personas y a 420 que pertenecen a una empresa contratista. Sin embargo, existen cuestionamientos sobre la integración de nuevos procesos de producción y gestión, que ya tienen diez ańos desde su aplicación y aún existe dificultad para conseguir los logros esperados a nivel organizacional e individual.

\section{Planteamiento del problema de estudio}

El sector minero no es ajeno a los cambios que los avances tecnológicos, los nuevos procesos productivos y la incorporación de maquinarias y tecnología cada vez más compleja. Además, la implementación de nuevos procedimientos de gestión orientados a la optimización de sus procesos y obtención de mejores resultados económicos, impacta a la organización minera en su totalidad, exigiendo una constate disposición y flexibilidad para el cambio e innovación tecnológica por pare de sus miembros. Considerando lo anterior, el objetivo 
del estudio es investigar la relación entre la actitud y la resistencia hacia el cambio organizacional en trabajadores de una empresa del rubro de la minería, partiendo del supuesto que los trabajadores que tienen una actitud favorable al cambio presentarán baja resistencia al cambio organizacional. El planteamiento de este problema de investigación se basa en los resultados de varios estudios, donde se encontraron correlaciones significativas entre la dimensión de temor al cambio organizacional y las variables de participación (integración), comunicación, trabajo en equipo, aceptación, conocimiento de la organización, cultura de cambio, proyección y desarrollo del cambio (Rubiano, Rojas \& Díaz, 2011). Otros estudios muestras que la falta de canales de comunicación entre la gerencia y los empleados es lo que produce mayor resistencia al cambio (Arras Vota, 2008; García Rubiano, 2011; Mazzei, 2014). Finalmente, un último estudio realizado con el propósito de determinar la relación entre la percepción de la justicia organizacional y cambio organizacional, mostró una relación significativa entre ambas variables, evidenciando la necesidad de considerarlo en la toma de decisiones y diseño de políticas de gestión del talento humano en función del cambio de la cultura organizacional a la que se quiere llegar (García \& Forero, 2014).

\section{Método}

El estudio corresponde a una investigación empírica y se utiliza una estrategia asociativa de tipo transversal y un diseño de grupos naturales (Ato, López \& Benavente, 2013).

\section{Participantes}

La muestra es probabilística de tipo estratificado. Los participantes fueron 251 trabajadores. Un 10.8\% son mujeres y el 89.2\% son hombres, con edades en los rangos de entre 18 a 30 ańos (22.3\%), 31 a 40 años (82\%), 41 a 50 años (26.3\%) y de 51 años o más (18.7\%). El grupo de trabajadores se compone por gerente/superintendente 
(3.2\%), supervisores $(16.7 \%)$, profesionales $(8.4 \%)$, técnicos $(10.4 \%)$ y administrativos $(61.4 \%)$, además están distribuidos en cinco faenas de producción, 1(61\%), 2(6\%), 3(12.4\%), 4(13.1\%) y 5(7.6\%). Respecto a la antigüedad laboral se distribuyen de la siguiente manera, menos de 2 años (26.7\%), de 2 a 10 años (55.4\%), más de 11 años (18\%) (ver Tabla 1).

\section{Tabla 1}

Variables sociodemográficas y factores en Estudio

\begin{tabular}{llcl}
\hline Variables & Categoría & Frecuencia & $(\%)$ \\
\hline \multirow{2}{*}{ Sexo } & Masculino & 224 & 89.2 \\
& Femenino & 27 & 10.8 \\
\hline \multirow{4}{*}{ Edad } & Entre 18 y 30 años & 56 & 22.3 \\
& Entre 31 y 40 años & 82 & 32.7 \\
& Entre 41 y 50 años & 66 & 26.3 \\
& 51 años o más & 47 & 18.7 \\
\hline \multirow{5}{*}{ Faena laboral } & Faena 1 & 153 & 61 \\
& Faena 2 & 15 & 6 \\
& Faena 3 & 31 & 12.4 \\
& Faena 4 & 33 & 13.1 \\
& Faena 5 & 19 & 7.6 \\
\hline \multirow{5}{*}{ Jerarquía en la } & Gerente-Superintendente & 8 & 3.2 \\
organización & Supervisor & 42 & 16.7 \\
& Profesional & 21 & 8.4 \\
& Técnico & 26 & 10.4 \\
& Operador & 154 & 61.4 \\
\hline \multirow{5}{*}{ Antigüedad laboral } & Menos de 2 años & 67 & 26.7 \\
& Entre 2 y 5 años & 81 & 32.3 \\
& Entre 6 y 10 ańos & 58 & 23.1 \\
& Entre 11 y 15 años & 16 & 6.4 \\
& 15 años o más & 29 & 11.6 \\
\hline
\end{tabular}




\section{Medición}

Escala de Actitudes hacia el Cambio (EAC): Se utilizó la escala desarrollada por Rabelo, Ross y Torres da Paz (2004). La EAC cuenta con una categoría que describe las creencias, comportamientos y sentimientos desarrollados por los individuos. La categoría principal es actitud frente al cambio, que cuenta con 50 ítems distribuidos en tres subcategorías: actitud de cinismo frente al cambio $(2,4,5,7,10,14$, $15,18,36,37,42,43,44)$; actitudes de temor ante el cambio $(6,9$, $13,16,17,19,21,22,23,25,29,32,33,34)$ y actitud de aceptación ante el cambio $(1,4,8,11,12,20,24,26,27,28,30,31,35,38,39$, $40,41,45,46,47,48)$. Fue medida mediante una escala Likert de 0 a 4 (donde $0=$ muy en desacuerdo y 4 = totalmente de acuerdo) (García et al., 2011). Esta escala muestra buenos niveles de fiabilidad y validez para ser utilizada en las investigaciones sobre los determinantes o las consecuencias del cambio organizacional. Su estructura factorial de tres factores, Aceptación del Cambio $(\alpha=.95)$, Temores ante el Cambio $(\alpha=.88)$ y Cinismo $(\alpha=.84)$ tiene buenos índices de consistencia interna y de validez de constructo (Rabelo, Ros \& Torres, 2004).

Escala de Resistencia al Cambio $\left(\mathrm{ERC}^{6}\right)$. Es una escala desarrollada por García (2005). Este instrumento está conformado por una serie de factores con escala cualitativa (excelente, muy bueno, normal, con fallas, inadecuados) y un baremo cuantitativo que va de 0 a 12 , el baremo cuantitativo guarda estrecha relación con la forma de presentar la información mediante gráficos radiales con características similares al de telaraña. Pueden identificarse seis segmentos diferenciados en la escala cuantitativa que también facilita el trabajo de la interpretación, procesamiento y presentación de los resultados, pues pueden ser asociados a las zonas de resistencia, neutra o flexibilidad. Este instrumento consta de seis partes, tres asociados a los factores individuales y los otros tres restantes a los factores de la organización: a) Diagnóstico de factores Individuales asociados al Saber/Conocer; b) Diagnóstico de factores Individuales asociados a la Responsabilidad (hacer); c) Diagnóstico de

6 Abreviación de los autores. 
factores Individuales asociados al Querer/Sentir/Desear; d) Diagnóstico de Factores asociados a la Organización; e) Diagnóstico de Factores Organizacionales asociados a los stakeholders (involucrados) - Afectado y f)Diagnóstico de Factores Organizacionales asociadas al Proyecto (Cambio). Esta escala presenta niveles adecuados de confiabilidad y validez $(\alpha=.84)$ (García, Mónica, Paola \& Bravo, 2007).

Encuesta sociodemográfica para recabar datos relacionados con la faena (lugar) laboral donde se desempeńa, el nivel jerárquico que ocupa dentro de la organización, el rango de antigüedad en años que ha permanecido en la organización y el rango etario en el cual se ubica. Con este instrumento se puede estratificar las diferentes categorías que tiene la empresa.

\section{Procedimiento}

Primeramente, se presentó el proyecto de investigación al gerente general a través de la Gerencia de Gestión de Personas, el cual accedió a lo solicitado, dejando el compromiso de entregar toda la información obtenida a la empresa y resguardando la confiabilidad de los procesos. En esa instancia se entregó los registros de las faenas para poder dar inicio al proceso de aplicación de instrumentos a los trabajadores. Con la aprobación, se da inicio a la etapa de aplicación de los instrumentos. Los trabajadores fueron convocados a salones próximos a su lugar de trabajo donde se les proporcionó los objetivos de la administración de los cuestionarios y se recalcó el carácter de confidencialidad de la información asegurando el buen uso de los datos. Cabe destacar que el presente estudio fue revisado y aprobado por un Comité de Ética Científica de la institución de los autores. Los estudiantes no recibieron ningún incentivo por participar y se tomaron todos los resguardos para garantizar el cumplimiento de los aspectos éticos de la investigación con seres humanos propuestos en las directrices de la American Psychological Association.

\section{Análisis de datos}

Para el procesamiento de los datos se utilizó el software SPSS 22.0. Para describir las diferentes dimensiones de la actitud y resistencia al 
cambio se calcularon los promedios y desviaciones estándar, así también las correlaciones lineales de Pearson $(r)$ entre el total de la escala de actitud (EAC) y la resistencia al cambio (ERC), y en cada una de las dimensiones que la componen (Nunnally, 1978). Las diferencias se estimaron utilizando el análisis de varianza (ANOVA) de un factor en función de las variables sociodemográficas (rango etáreo, años de antigüedad, faena laboral y jerarquía en la organización). Para el sexo se utilizó la prueba $t$ de Student para muestras independientes. En el caso de no existir homogeneidad, se aplicó el test de Welch (Armitaje, Berry \& Matthews, 1994). Las comparaciones post-hoc se realizaron aplicando la prueba de Tukey y Bonferroni. Se utilizó como medida del tamaño del efecto la $d$ de Cohen y Eta parcial al cuadrado $\left(\eta_{p}\right)$ (Cohen, 1988).

\section{Resultados}

\section{Datos descriptivos de Actitudy Resistenciaal Cambio Organizacional}

Los datos indican una actitud favorable hacia el cambio organizacional de parte de los participantes $(M=113.7, D E=23.17)$. Sin embargo, se presenta una resistencia al cambio media/moderada $(M=$ 185.9, $D E=27.28)$. Los valores más altos obtenidos con el ERC se ubican en las dimensiones Factores Individuales Asociados a Desear el Cambio $(M=50.98, D E=8.29)$ y Factores Individuales Asociados al Saber $(M=30.49, D E=5.01)$, y las puntuaciones más bajas corresponden a Factores Organizacionales Asociados a los Otros $(M=12.48$, $D E=3.22)$ y Factores Organizacionales Asociados a los Agentes de Cambio $(M=12.6, D E=3.13)$. El valor más alto obtenido con el EAC corresponde a la dimensión Actitud de Aceptación del Cambio $(M=55.86, D E=10.6)$, y la puntuación más baja a Actitud de Cinismo al Cambio $(M=27.23, D E=6.33)$.

Como se presenta en la Tabla 2, las correlaciones más fuertes se observan entre Actitud de Cinismo al Cambio y; Factores Individuales Asociados a la Responsabilidad $r(251)=-.27, p=.00$; y Factores Individuales Asociados al Desear $r(251)=-.31, p=.00$. Así también, 
Actitud de Temor al Cambio y Factores organizacionales asociados al proceso de cambio $r(251)=-.25, p=.000$. Por último, existe correlación negativa entre las puntuaciones totales de las Escalas de Actitud (EAC) y Resistencia al Cambio Organizacional (ERC) $r(251)=-.14$, $p=.03$.

\section{Tabla 2}

Correlaciones entre las Dimensiones de Actitud y Resistencia al Cambio

\begin{tabular}{|c|c|c|c|c|c|c|c|c|c|c|}
\hline \multirow{2}{*}{$\begin{array}{l}\text { Dimensiones } \\
\text { EAC }\end{array}$} & \multicolumn{10}{|c|}{ Dimensiones ERC } \\
\hline & 1 & 2 & 3 & 4 & 5 & 6 & 7 & 8 & 9 & 10 \\
\hline ACC (1) & 1 & $.61^{* *}$ & $.50^{* *}$ & -.12 & $-.27^{* *}$ & $-.31^{* *}$ & $-.19^{* *}$ & -.03 & -.11 & $-.20^{* *}$ \\
\hline ATC (2) & & 1 & $.66^{* *}$ & -.03 & -.11 & $-.18^{* *}$ & $-.25^{* *}$ & .03 & -.06 & $-.17^{* *}$ \\
\hline $\mathrm{AAC}(3)$ & & & 1 & .10 & .02 & .01 & -.08 & .12 & .09 & .00 \\
\hline FIAS (4) & & & & 1 & $.48^{* *}$ & $.55^{* *}$ & $.38^{* *}$ & $.18^{* *}$ & $.21^{* *}$ & $.38^{* *}$ \\
\hline FIAR (5) & & & & & 1 & $.53^{* *}$ & $.30^{* *}$ & $.17^{* *}$ & $.22^{* *}$ & $.29^{* *}$ \\
\hline FIAD (6) & & & & & & 1 & $.54^{* *}$ & $.32^{* *}$ & $.38^{* *}$ & $.36^{* *}$ \\
\hline FOAPC (7) & & & & & & & 1 & $.39^{* *}$ & $.41^{* *}$ & $.62^{* *}$ \\
\hline FOAO (8) & & & & & & & & 1 & $.79^{* *}$ & $.38^{* *}$ \\
\hline FOAAC (9) & & & & & & & & & 1 & $.47^{* *}$ \\
\hline FOAPR (10) & & & & & & & & & & 1 \\
\hline
\end{tabular}

${ }^{* *} p<.01$; ACC=Actitud de cinismo al cambio; ATC=Actitud de temor al cambio; AAC= Actitud de aceptación al cambio, FIAS=Factores individuales asociados al saber; FIAR=Factores individuales asociados a la responsabilidad; FIAD $=$ Factores individuales asociados al desear; FOAPC=Factores organizacionales asociados al proceso de cambio; $\mathrm{FOAO}=$ Factores organizacionales asociados a los otros; FOAAC=Factores organizacionales asociados a los agentes de cambio; FOAPR=Factores organizacionales asociados al proyecto.

\section{Diferencias en Actitud y Resistencia al Cambio en Función de las variables Sociodemográficas y Sociolaborales}

Respecto a la actitud y resistencia al cambio en función del sexo, las mujeres presentan puntuaciones más altas que los hombres en los Factores Individuales Asociados al Desear (ver Tabla 3), siendo el tamaño del efecto moderado en las diferencias de la dimensión FIAD. 


\section{Tabla 3}

Diferencias en las Dimensiones de ERC y EAC en función del sexo

\begin{tabular}{lcccccc}
\hline \multirow{2}{*}{$\begin{array}{l}\text { ERC/EAC } \\
\text { Dimensiones }\end{array}$} & \multicolumn{2}{c}{ Media $(D E)$} & $g l$ & $t$ & $p$ & $d$ \\
\cline { 2 - 3 } ERC & Femenino & Masculino & & & & \\
FIAS & $31.48(5.26)$ & $30.37(4.98)$ & 251 & -1.09 & .28 & \\
FIAR & $17.04(2.24)$ & $15.83(3.13)$ & 251 & -1.95 & .053 & \\
FIAD & $55.37(9.74)$ & $50.45(7.96)$ & 251 & -2.956 & .00 & .55 \\
FOAPC & $30.63(7.05)$ & $29.08(6.71)$ & 251 & -1.12 & .26 & \\
FOAO & $13.07(2.44)$ & $12.40(3.30)$ & 251 & -1.02 & .31 & \\
FOAAC & $13.11(2.60)$ & $12.54(3.19)$ & 251 & -.90 & .37 & \\
FOAPR & $34.07(8.76)$ & $33.87(8.40)$ & 251 & -.12 & .91 & \\
EAC & & & & & & \\
ACC & $25.78(6.58)$ & $27.4(6.29)$ & 251 & 1.26 & .21 & \\
ATC & $30.00(10.23)$ & $30.68(9.92)$ & $32.067^{*}$ & .33 & .75 & \\
AAC & $56.30(13.16)$ & $55.81(10.28)$ & 251 & -.23 & .82 & \\
\hline
\end{tabular}

*Dado que las varianzas son desiguales, se aplicó el test de Welch.

Nota. FIAS=Factores individuales asociados al saber; FIAR=Factores individuales asociados a la responsabilidad; $\mathrm{FIAD}=$ Factores individuales asociados al desear; FOAPC=Factores organizacionales asociados al proceso de cambio; $\mathrm{FOAO}=$ Factores organizacionales asociados a los otros; FOAAC=Factores organizacionales asociados a los agentes de cambio; FOAPR=Factores organizacionales asociados al proyecto; $\mathrm{ACC}=$ Actitud de cinismo al cambio; $\mathrm{ATC}=$ Actitud de temor al cambio y $\mathrm{AAC}=$ Actitud de aceptación al cambio.

En relación con la faena de trabajo (ver Tabla 4), se observan diferencias en la Escala de Resistencia al Cambio (ERC), siendo el efecto de está en las diferencias de pequeño a moderado, $F(4,242)=2,70, p<$ $.05, \eta_{\mathrm{p}}^{2}=.04,95 \%$ ICs $[177.19,185.91],[187.05,206.28],[179.71$, 206.02], [182.38, 198.59] y [182.40, 204.65], respectivamente. Así también, se presentan diferencias significativas en las siguientes dimen- 
siones del ERC; FIAS, $F(4,246)=4.04, p<.01, \eta_{\mathrm{p}}{ }^{2}=.06,95 \%$ ICs [28.84, 30.50], [30.58, 35.42], [30.90, 34.97], [29.55, 32.27] y [28.99, 31.64], respectivamente; FIAR, $F(4,246)=4.38, p<.01, \eta_{\mathrm{p}}{ }^{2}=.06,95 \%$ ICs $[14.93,15.91],[17.27,19.39],[15.34,18.01],[15.79,17.18] \mathrm{y}$ $[14.74,17.89]$, respectivamente y; FIAD, $F(4,244)=4.41, p<.01, \eta_{\mathrm{p}}{ }^{2}$ $.06,95 \%$ ICs $[48.09,50.66],[54.03,58.51]$, [49.97, 56.70], [49.62, $56.32]$ y $[49.46,55.59]$, respectivamente. En todos los casos el tamaño del efecto en las diferencias fue medio/moderado. Además, se presentan diferencias significativas en la Escala de Actitud hacia el Cambio (EAC) en función de la faena laboral, $F(4,245)=3.72, p<.01, \eta_{\mathrm{p}}{ }^{2}=.05$, 95\% ICs [111.19, 118.61], [93.55, 117.12], [117.04, 131.86], [97.70, $114.42]$ y $[96,11,117.26]$, respectivamente, también se observan diferencias significativas en las siguientes dimensiones del EAC; Actitud de Cinismo al Cambio, $F(4,246)=5,63, p<.001, \eta_{\mathrm{p}}{ }^{2}=.08,95 \%$ ICs [27.20, 29.22], [20.27, 20.07], [26.44, 31.24], [22.77, 26.68] y $[21.82,25.86]$, respectivamente, y; Actitud de Temor al Cambio, $F(4,246)=3.20, p<.05, \eta_{\mathrm{p}}{ }^{2}=.04,95 \%$ ICs [29.23, 32.45], [23.40, $32.20]$, [32.15, 38.43], [23.53, 30.89] y [24.78, 33.64], respectivamente. El tamaño del efecto de la faena laboral en las diferencias fue pequeńo en la EAC y ATC, y moderado en la dimensión ACC.

Con relación a la Jerarquía Laboral (ver Tabla 5), se observan diferencias en las siguientes dimensiones del ERC; FIAS, $F(4,246)=3.78$, $p<.01, \eta_{p}{ }^{2}=.06,95 \%$ ICs [28.62, 33.63], [31.39, 33.85], [30.06, $33.65]$, [29.37, 32.71] y [28.73, 30.45], respectivamente; FIAR, $F(4$, $246)=5.85, p<.001, \eta_{p}=.08,95 \%$ ICs $[16.78,19.72]$, [16.61, 17.96], $[15.22,17.55],[15.74,17.72]$ y $[14.76,15.81]$, respectivamente y; FIAD, $F(4,244)=3.94, p<.01, \eta_{p}=.06,95 \%$ ICs [50.83, 58.42], $[52.43,55.71],[51.12,55.78],[49.32,54.99]$ y [47.94, 50.90], respectivamente para cada Jerarquía Laboral. En todos los casos el tamaño del efecto en las diferencias fue moderado. 


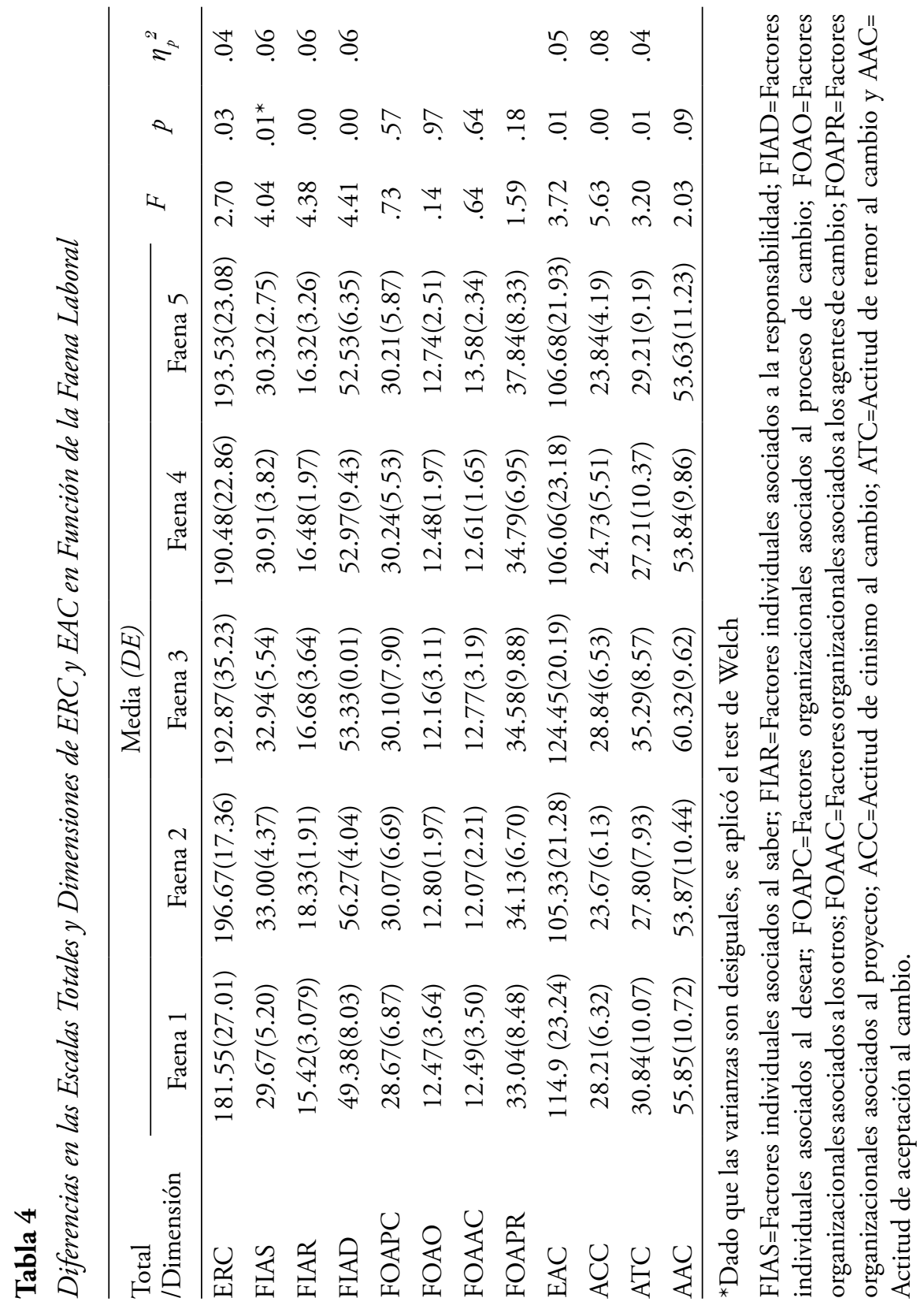




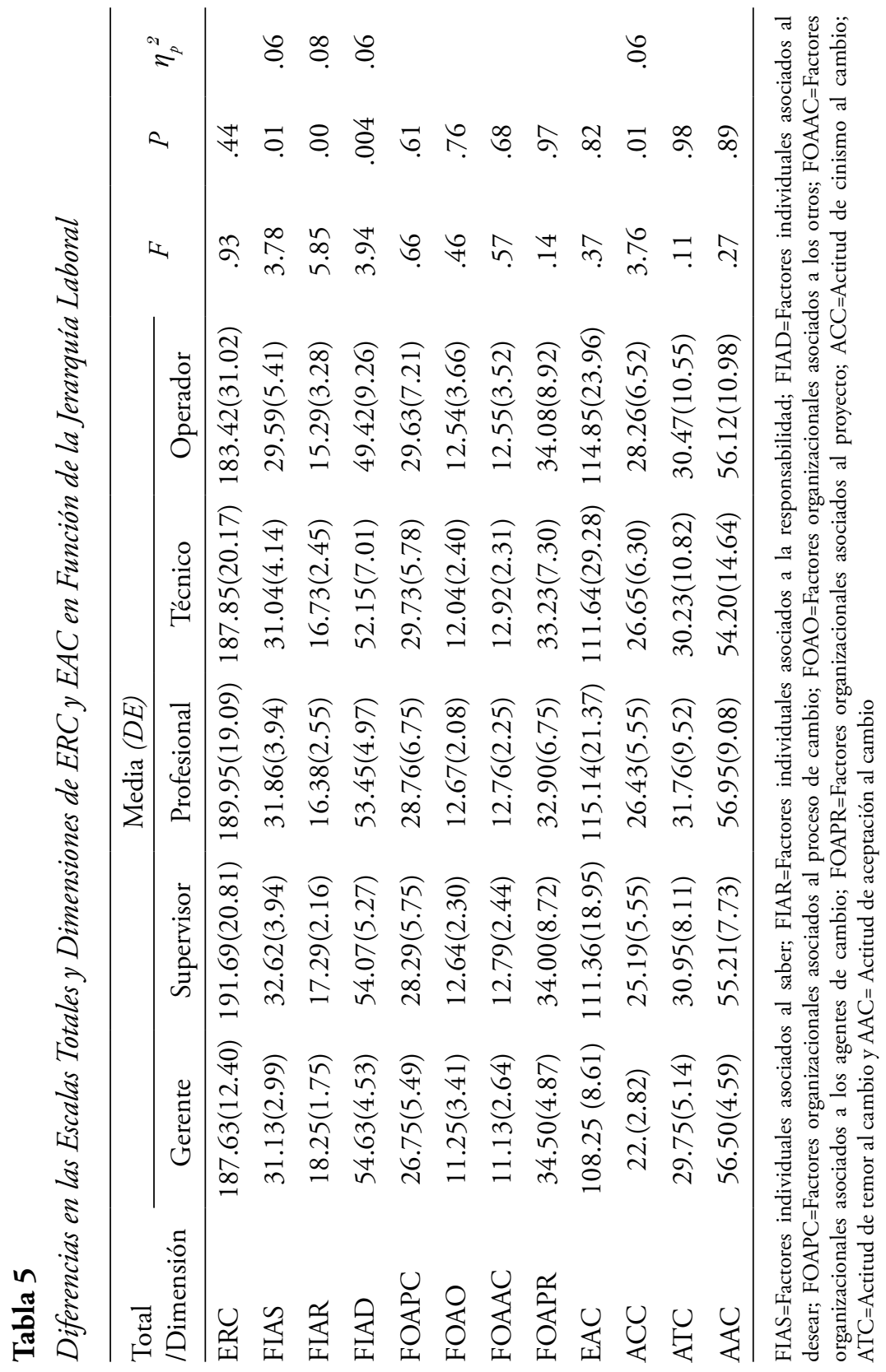


Respecto a la EAC, se presentan diferencias en la dimensión Actitud de Cinismo al Cambio en función de la Jerarquía Laboral, $F(4,244)=3.76, p<.01, \eta_{\mathrm{p}}{ }^{2}=.06,95 \%$ ICs [19.64, 24.36], [23.46, 26.92], [23.90, 28.96], [24.11, 29.20] y [27.22, 29.30], respectivamente. El tamańo del efecto en las diferencias fue moderado.

Respecto a la Edad del trabajador la única diferencia encontrada es en la dimensión Actitud de Temor Hacia el Cambio, F $(3,247)=3.23$, $\mathrm{p}<.05,95 \%$ ICs [25.96, 31.83], [31.31, 35.08], [28.13, 32.63] y [25.17, 31.76], respectivamente, siendo el rango de "51 años o más" el que presenta un promedio menor $(\mathrm{M}=28.47, \mathrm{DE}=11.22)$, y la categoría "entre 31 a 40 ańos" obtiene la media mayor $(\mathrm{M}=33.20, \mathrm{DE}=$ 8.55) El tamaño del efecto en las diferencias es pequeño. Con relación a la Antigüedad Laboral no se presentan diferencias en las escalas ERC y EAC, y las dimensiones que la componen.

\section{Discusión}

El objetivo de la investigación fue analizar la relación entre la actitud y resistencia al cambio organizacional en trabajadores mineros de una empresa ubicada en la región de Atacama, Chile, la cual incorpora recurso humano que se desempeña en un contexto de elevada exigencia, y que actualmente se enfrenta a constantes cambios y reestructuraciones debido a las fluctuaciones propias del valor del cobre. Los resultados dan a conocer una actitud positiva hacia el cambio organizacional, sin embargo, una mediana resistencia al mismo. Si bien esto pareciera ser contradictorio, las dimensiones Actores Organizacionales Asociados a los Otros y Agentes de Cambio puntúan más bajo, lo que significa que la resistencia estaría asociada a una percepción negativa de los factores de tipo externos y no individuales. Lo anterior, fue confirmado por el estudio de Rodríguez y Mladinic (2016), quienes plantean los trabajadores "indiferentes" o "medianamente resistentes" son actores claves para una mejor comprensión de los procesos psicológicos involucrados en las respuestas de las personas cuando se ven enfrentadas a modificaciones en sus contextos laborales. 
Por otra parte, las puntuaciones más altas en la ERC y EAC, se ubican en dos dimensiones: 1) Factores Individuales Asociados a Desear el Cambio y al Saber; y 2) en la dimensión Actitud de Aceptación del Cambio, respectivamente. Las correlaciones más fuertes, e indirectas, se observan entre Factores Individuales Asociados a la Responsabilidad y Factores Individuales Asociados al Desear con la Actitud de Cinismo al Cambio. Así también, se presenta similar relación entre Factores organizacionales asociados al proceso de cambio y Actitud de Temor al Cambio. Lo anterior es posible asociar a los planteamientos de Rubiano, Rojas y Díaz (2011), quienes sugieren que la dimensión de Actitud de Cinismo al Cambio debe correlacionar en dirección negativa con los Factores Individuales y Factores Organizacionales. Por otra parte, la dimensión Actitud de Temor al Cambio y los Factores Organizacionales Asociados al Proceso de cambio, se relacionan en este estudio indirectamente, consistente con lo planteado por Lefcovich (2006), quien afirma que entre las principales causas asociadas a la resistencia al cambio se encuentran el temor a lo desconocido y el clima de baja confianza en la organización entre otros.

Respecto a las jerarquías laborales, los gerentes y supervisores perciben positivamente sus destrezas y competencias individuales para contribuir con el cambio, orientando sus acciones hacia la comprensión y el cumplimiento de las responsabilidades básicas, generando también sentimientos y deseos tendientes hacia el proceso de cambio organizacional. Así, Van Dam y colaboradores (2008), han constatado que la relación entre jefaturas y trabajador es una variable crucial en la promoción del cambio. En el presente estudio los trabajadores involucrados en procedimientos de producción como profesionales, técnicos y operadores de maquinaria presentaron una actitud propensa de cinismo al cambio, lo que se traduce en una percepción de desconfianza frente a las capacidades de los agentes precursores del cambio, es decir las jefaturas. Lo anterior se apoya en lo planteado por Giangreco y Peccei (2005, como se citó en Erwin \& Garman, 2010), quienes afirman que la participación en el proceso de cambio está asociado a la percepción positiva del mismo. 
Cabe destacar que los trabajadores de mayor edad (50 años o más) presentan menor Actitud de Temor hacia el Cambio, que aquellos que se ubican en el rango entre 31 a 40 ańos. Por otra parte, se observan diferencias significativas respecto de la Actitud hacia el Cambio Organizacional entre faenas de trabajo, correspondiendo los puntajes extremos a dos de estas. Aquella faena que presenta una actitud favorable ante el cambio, se caracteriza por trabajar según el Modelo de Excelencia Operacional (MEXO), que es un estándar implementado hace tres años en la empresa con el objetivo de reducir costos y acrecentar la productividad. Esta faena ha sido reconocida en la organización por la utilización de esta herramienta, siendo ellos los mentores para las otras secciones laborales.

Por otra parte, la faena que presenta una actitud negativa ante el cambio organizacional, no ha implementado adecuadamente el modelo de la empresa y ha disminuido notablemente la producción, lo que se podría asociar a lo planteado por Tornay y Milán (2001), quienes afirman que la evidencia empírica al respecto sugiere que cuando los miembros de una organización perciben que deben cambiar de una actividad a otra, la productividad tiende a decrecer, percibiéndose como amenazante - y por lo tanto - con la tendencia a ser la situación evaluada de forma negativa. En este contexto, se plantea como una importante línea de investigación a seguir, la preparación y/o apresto al cambio (Gagnon et al., 2014); con la cual se podría dar cuenta de las variables psicológicas previas, promotoras y/o inhibidoras del cambio organizacional, por ejemplo, cuando se activan los mecanismos de alerta cognitiva generadoras de respuestas evitativas de huida frente a la inestabilidad, perdida de la seguridad en el empleo e inminente cambio organizacional (Biggane, Allen, Amis, Fugate \& Steinbauer, 2017).

Por último, cabe señalar que las limitaciones del estudio se presentan fundamentalmente en el diseño de tipo transversal utilizado, por lo que se sugiere realizar a futuro investigaciones de tipo longitudinal que permitan visibilizar la relación temporal entre las variables estudiadas, y además incorporar instrumentos diseńados para aplicarse en el sector de la minería que permitan recopilar información sobre 
riesgo psicosocial, estrategias de afrontamiento al cambio organizacional y bienestar subjetivo del trabajador, con el fin de diseñar un modelo de carácter predictivo con mayor capacidad de generalización.

\section{Referencias}

Acosta, C. (2003). Paradoja de cambio organizacional de M.E. Malott. Revista Latinoamericana de Psicología, 35(1), 100-103. Recuperado de: http://www.redalyc.org/articulo.oa?id=80535113

Aldana de Becerra, G.M. \& Joya, N.S. (2011). Actitudes hacia la investigación cientifica en docentes de metodología de investigación. (U. C. Cundimarca, Ed.). Redalyc. Org (Tabula Rasa), 14, $295-$ 309. Recuperado de: http://www.scielo.org.co/pdf/tara/n14/ n14a12.pdf

Arras Vota, A.M. (2008). Comunicación y cambio organizacional. Revista Latina de Comunicación Social, 63, 418-434. https://doi. org/10.4185/RLCS-63-2008-792-418-434

Ato, M., López, J. \& Benavente, A. (2013). Un sistema de clasificación de los diseños de investigación en psicología. Anales de Psicología, 29(3), 1038-1059. https://dx.doi.org/10.6018/ analesps.29.3.178511

Benoit, G. \& Meston, F. (1995). Reingeniería del Cambio. diez claves para transformar la empresa (Vol. $\left.\mathrm{N}^{\circ} 10\right)$. (Marcombo, Ed.) México.

Biggane, J.E., Allen, D.G., Amis, J., Fugate, M. \& Steinbauer, R. (2017). Cognitive Appraisal as a Mechanism Linking Negative Organizational Shocks and Intentions to Leave. Journal of Change Management, 17(3), 1-25. https://dx.doi.org/10.1080/1 4697017.2016.1219379

Chiavenato, I. (2004). Introducción a la teoría general de la administración (7a edición). México D.F.: Mc. Graw Hill.

Chiavenato, I. (2009). Comportamiento Organizacional (2a edición). (J.M. Chacón, Ed.) México D.F.: Mc Graw Hill. 
Chiavenato, I. (2011). Administración de Recursos Humanos (9a edición). (J.M. Chacón, Ed.) México, D.F., México: McGraw-Hill Interamericana editores S.A. de C.V.

Chreim, S. (2006). Managerial frames and institutional discourses of change: employee appropriation and resistance. Organization Studies, 27(9), 61-87. https://doi.org/10.1177/0170840606064106

Cloninger (2003). La Perspectiva de los Rasgos. En S. C. Cloninger, Teorías de la Personalidad (A. F. Salinas, Trad., Tercera Edición ed., pág. 592). México, México: Prentice Hall.

Cohen, J. (1988). Statistical power analysis for the behavioral sciences (2nd ed.). Hillsdale, NJ: Lawrence Earlbaum Associates.

Comisión Chilena del Cobre [Cochilco] (n.d.). Minería en Chile: Impacto en Regiones y Desafíos para su Desarrollo. Ministerio de Minería de Chile. Recuperado de: https://www.cochilco.cl/ Libros/Libro_Mineria_en_Chile_Impacto_en_Regiones_y_ Desafios_para_su_Desarrollo.pdf

Contreras, F. \& Barbosa, D. (2013). Del liderazgo transaccional al liderazgo transformacional: implicaciones para el cambio organizacional. Revista virtual universidad católica del norte, 39, 153-164. http://www.redalyc.org/pdf/1942/194227509013.pdf

De Mazzei, S.Z. (2014). Resistencia al cambio organizacional en una pequeña y mediana empresa (PyME) del sector industrial. Revista Telos, 16(2), 226-242. Recuperado de: http://google. redalyc.org/articulo.oa?id $=99331125002$

Dennis, E. \& Garman, A. (2010). Resistance to organizational change: linking research and practice. Leadership \& Organization Development Journal, 31(1), 39-56. https://dx.doi. org/10.1108/01437731011010371

DiFabio, A. \& Gori, A. (2016). Developing a New Instrument for Assessing Acceptance of Change. Front. Psychol, 7, 1-10. https:// doi.org/10.3389/fpsyg.2016.00802

DiFabio, A. \& Kenny, M.E. (2015). The contributions of emotional intelligence and social support for adaptive career progress 
among Italian youth. J.Career Dev, 42(1), 48-49. https://doi. org/10.1177/0894845314533420

DiFabio, A. \& Saklofske, D.H. (2014). Comparing ability and selfreport trait emotional intelligence, fluid intelligence, and personality traits in career decision. Pers.Individ.Dif. 64, 174178. https://doi.org/10.1016/j.paid.2014.02.024

DiFabio, A. \& Bucci, O. (2015). Affective profiles in Italian highschool students: Life satisfaction, psychological well-being, self-esteem, and optimism. Front. Psychol, 6, 1310, 1-6. https:// doi.org/10.3389/fpsyg.2015.01310

Erwin, D. \& Garman, A. (2010). Resistance to organizational change: linking research and practice. Leadership of Organization Development Journal, 31(1), 39-56 https://dx.doi. org/10.1108/01437731011010371

Ferrer, R.M. (2015). La influencia del factor humano, el liderazgo y la cultura de las organizaciones en los procesos de implementación y gestión del cambio organizacional. Revista Internacional de Investigación en Ciencias Sociales, 11(1), 102-114. https://dx.doi. org/10.18004/riics.2015.julio.102-114

Foldal, V, Langvik E. \& Saksvik-Lehouillier, I. (2016). Dispositional factors and work mastery among shift workers. Chronobiol Int. 3(6), 650-652. https://dx.doi.org/10.3109/07420528.201 6.1167718

Furnham, A. (2001). Psicología organizacional. México: Oxford University.

Gagnon, M.P., Attieh, R., Ghandour, E.K., Le gare, F., Ouimet, M, Estabrooks, C. \& Grimshaw, J. (2014) A Systematic Review of Instruments to Assess Organizational Readiness for Knowledge Translation in Health Care. PLoS ONE, 9(12), e114338. https:// dx.doi.org/10.1371/ journal.pone.0114338

García, C.G. (2005). Herramienta para el Diagnostico de la Resistencia al Cambio durante el desarrollo de Proyectos Mayores. (E. Gerenciales, Ed.) Redalyc. Org. Red de Revistas Cientificas de America Látina, El Caribe, España y Portugal (96), 57-106. 
Recuperado de http://www.scielo.org.co/pdf/eg/v21n96/ v21n96a03.pdf

García R, Mónica R, Rubio, P. \& Bravo, L. (2007). Relación entre los factores de riesgo psicosocial y la resistencia al cambio organizacional en personal de seguridad de una empresa del sector económico terciario. Diversitas, 3(2), 301-315. Recuperado de http://pepsic.bvsalud.org/scielo.php?script=sci_ arttext\&pid=S1794-99982007000200011\&lng=pt\&tln $\mathrm{g}=\mathrm{es}$.

García Rubiano, M. (2011). Liderazgo transformacional y la facilitación de la aceptación al cambio organizacional. Pensamiento Psicológico,9(16), 41-54. Recuperadodehttp://www.scielo.org.co/scielo. php?script=sci_arttext\&pid=S1657-89612011000100004\&lng $=$ en\&tlng=es

García, M. \& Forero, C. (2014). Cambio organizacional y percepción de la justicia organizacional en una entidad de la ciudad de Bogotá-Colombia. Revista diversitas - Perspectivas en psicología, 10(2), 293-405. Recuperado de http://www.scielo.org.co/pdf/ dpp/v10n2/v10n2a08.pdf

García, M., Rojas, M.F. \& Díaz, S. (2011). Relación entre el cambio organizacional y la actitud al cambio de una empresa del sector terciario. (e.C. Red de Revistas Científicas de América Latina, Ed.) Diversitas: Perspectivas en Psicología, 7(1), 125-142. Recuperado de http://www.scielo.org.co/scielo.php?script=sci_arttex t\&pid=S1794-99982011000100009

García, M., Gómez, G. P. \& Londoño, P.I. (2009). Relación entre motivación y resistencia al cambio en personas que trabajan en una empresa del sector público, en Bogotá (Colombia). Revista Diversitas Perspectivas en Psicología, 5(1), 141-159. Recuperado de http://www.redalyc.org/pdf/679/67916259012.pdf

García, M. \& Forero, C. (2010). IMC-Instrumento de Medición de Cambio Organizacional. Manual Técnico. Bogotá, Colombia: RH\&T. (Documento no publicado). 
García-Rubiano, M., \& Forero-Aponte, C. (2015). Contrato psicológico y cambio organizacional en una entidad perteneciente al sector terciario de la ciudad de Bogotá, Colombia. Rev.investig.desarro. innov, 6(1), 15-28. https://doi.org/10.19053/20278306.4047

Gil, M. \& Caccioppo, J. (2007). Psicología Social, un compromiso aplicado a la salud ( $1^{\circ}$ edición ed.). (P. u. Zaragoza, Ed.) España: Universidad de Zaragoza.

Johansson, C., Åström, S., Kauffeldt, A., Helldin, L. \& Carlström, E. (2014). Culture as a predictor of resistance to change: a study of competing values in a psychiatric nursing context. Health Policy, 114(2-3), 156-62. https://dx.doi.org/10.1016/j. healthpol.2013.07.014

Lee, S.Y., Weiner, B.J., Harrison, M.I. \& Belden, C.M. (2013). Organizational transformation: a systematic review of empirical research in health care and other industries. Med Care Res Rev, 70(2), 115-42. http://dx.doi.org/10.1177/1077558712458539

Lefcovich, M. (2006). Superando la resistencia al cambio. [online]. Recuperado de http://www.degerencia.com/articulo/superando_ la_resistencia_al_cambio

López, M.E., Restrepo, L.E. \& López, G.L. (2013). Resistencia al cambio en organizaciones modernas. Scientia et Technica, 18(1), 149-157. Recuperado de http://www.redalyc. org/pdf/849/84927487022.pdf

Macrì, D.M., Tagliaventi, M.R. \& Bertolotti, F. (2002) Grounded theory for resistance to change in a small organization. Journal of Organizational Change Management, 15(3), 292-310. https:// dx.doi.org/10.1108/095348110210429327

Montealegre, J.V. \& Calderon, G.H. (2007). Relación entre actitud hacia el cambio y cultura organizacional (Innovar, Ed.). Revista Innovar, 17(29), 49-70. Recuperado de http://www.redalyc.org/ pdf/818/81802904.pdf

Nunnally, J. C. (1978). Psychometric Theory. New York: McGraw-Hill. Oreg, S. (2006). Personality, context, and resistance to organizational change. European Journal of Work and Organi- 
zational Psychology, 15(1), 73-101. https://dx.doi.org/10.1080/ 13594320500451247

Ossandón, J. (2013). Factores que obstaculizan el cambio en profesores y directivos de liceos vulnerables en la región de la Araucanía (Chile). EDUCAR, 492, 183-206. https://doi.org/10.5565/rev/ educar. 416

Packard, T. \& Shih, A. (2014) Organizational Change Tactics: The Evidence Base in the Literature, Journal of Evidence-Based Social Work, 11(5), 498-510. https://dx.doi.org/10.1080/15433714.2 013.831006

Pahkin, K., Nielsen, K., Vänänen, A., Mattila-Holappa, P., Leppänen, A. \& Koskinen, A. (2014). Importance of change appraisal for employee well-being during organizational restructuring: findings from the Finnish paper industryss extensive transition. Ind Health, 52(5), 445-55. https://dx.doi.org/10.2486/ indhealth.2014-0044

Pérez, L.M., Vilariño, C.M. \& Ronda, G.A. (2016). El cambio organizacional como herramienta para coadyuvar la implementación de la estrategia. Ingeniería Industrial, 37(3), 286-294. Recuperado de http://scielo.sld.cu/scielo.php?script= sci_arttext\&pid=S1815-59362016000300007\&lng=es\&tln $\mathrm{g}=\mathrm{es}$

Petrou, P., Demerouti, E. \& Schaufeli, W.B. (2016). Crafting the Change: The Role of Employee Job Crafting Behaviors for Successful Organizational Change. Journal of Management, 18, 1-27. https://dx.doi.org/10.1177/0149206315624961.

Porret, M.G. (2010). Gestión de personas, Manual para la gestión del capital humano en las organizaciones (Cuarta edición ed.). (ESIC, Ed.) Madrid, España.

Rabelo, E., Ros, M., Torres, M. (2004). Validación de una escala de actitudes ante el cambio organizacional. Revista de Psicología del Trabajo y de las Organizaciones, 20(1), 9-30. Recuperado de http://www.redalyc.org/articulo.oa?id=231317996002 
Rodríguez, M.D. (2011). Gestión Organizacional (Quinta edición ed.). (V. d. Continua, Ed.). Santiago, Chile: Ediciones Universidad Católica de Chile.

Rodríguez, V. \& Mladinic, A. (2016). Ambivalencia actitudinal ante el cambio organizacional: un análisis desde el individuo en contexto laboral. Psykhe (Santiago), 25(1), 1-17. https://dx.doi. org/10.7764/psykhe.25.1.733

Romero, J., Matamoros, S. \& Campo, C.A. (2013). Sobre el cambio organizacional. Una revisión bibliográfica. INNOVAR. Revista de Ciencias Administrativas y Sociales, 23(50), 35-52. Recuperado de http://www.redalyc.org/articulo.oa?id=81828692004

Rubiano, M., Rojas, M., Fernanda, M. \& Díaz, S. (2011). Relación entre el cambio organizacional y la actitud al cambio en trabajadores de una empresa de Bogotá. Diversitas: Perspectivas en Psicología, 7(1), 125-142. https://doi.org/10.15332/ s1794-9998.2011.0001.08

Schaffer, M.A., Sandau, K.E. \& Diedrick, L. (2013). Evidence-based practice models for organizational change: overview and practical applications. J Adv Nurs, 69(5), 1197-209. https://dx.doi. org/10.1111/j.1365-2648.2012.06122.x

Sánchez, J. \& Nguyen, O. (2009). Emotional Aperture and Strategic Change: The Accurate Recognition of Collective Emotions. Organizational Science, 20(1), 22-34. https://dx.doi. org/10.1287/orsc. 1070.0347

Sandoval, J.L. (2014). Los Procesos de Cambio Organizacional y la Generación de Valor. (B. C. Universidad ICESI, Ed.) Elsevier Doyma, 30(131), 162-171. https://doi.org/10.1016/j. estger.2014.04.005

Stefani, D. (2005). Teoria de Acción Razonada: Una propuesta de evaluación cuali-cuantitativa de las creencias acerca de la institucionalización geriátrica. Revista Universidad Nacional de Córdoba (Argentina), 5, 22-37. Recuperado de http://www.revistas.unc. edu.ar/index.php/revaluar/article/viewFile/538/478 
Tornay, F. \& Milán, E. (2001). Diferente participación de los mecanismos de control en el cambio entre tareas regular frente al cambio al azar. Psicothema, 13(1), 111-117. Recuperado de http://www.psicothema.es/pdf/421.pdf

Zuinaga de Mazzei, S. (2014). Resistencia al cambio organizacional en una pequeña y mediana empresa (PyME) del sector industrial. Telos, 16(2), 226-242. Recuperado de http://www.redalyc.org/ pdf/993/99331125002.pdf

Recibido: 9 de setiembre, 2016 Revisado: 29 de setiembre, 2017 Aceptado: 9 de octubre, 2017 